\title{
O ESTADO DA ARTE DA CROMATOGRAFIA LÍQUIDA DE ULTRA EFICIÊNCIA
}

\author{
Liane Maldaner e Isabel Cristina Sales Fontes Jardim* \\ Departamento de Química Analítica, Instituto de Química, Universidade Estadual de Campinas, CP 6154, 13084-971 \\ Campinas - SP, Brasil
}

Recebido em 8/4/08; aceito em 14/7/08; publicado na web 5/12/08

\begin{abstract}
THE STATE OF ART OF ULTRA PERFORMANCE LIQUID CHROMATOGRAPHY. Ultra Performance Liquid Chromatography (UPLC) retains the same principles as High Performance Liquid Chromatography (HPLC), but uses 1-2.1 mm i.d. columns with sub-2 $\mu \mathrm{m}$ particles. It is considered the newest advance in analytical separation science. The use of these small particles with mobile phases at high linear velocities increases resolution and detectability and decreases analysis time. Thus, the analyses are faster, the solvent volume is smaller, the efficiency is higher and the detectability is 2-3 times higher when compared with HPLC analysis.
\end{abstract}

Keywords: ultra performance liquid chromatography; sub-2 $\mu$ m particles; fast analysis.

\section{INTRODUÇÃO}

Desde o início da cromatografia líquida (CL), em 1950, até os dias atuais, muitos avanços foram alcançados e todos eles foram impulsionados pelo desenvolvimento contínuo de novas partículas de fases estacionárias (FE) que fossem capazes de gerar colunas mais seletivas, eficientes e estáveis química e mecanicamente. Nos últimos 40 anos, a cromatografia líquida de alta eficiência (CLAE) tem sido a técnica analítica mais desenvolvida, difundida e empregada em laboratórios de análise de indústrias químicas e farmacêuticas, em áreas médicas e em muitos outros campos da ciência e até em órgãos governamentais.

Associada a essa expansão, na última década, o desenvolvimento da CLAE tem sido direcionado à necessidade de análises mais rápidas, porém sem o comprometimento do desempenho cromatográfico. Para isso, a redução do tamanho das partículas da FE e das colunas foi a alternativa mais atrativa, porém ficou limitada por um período por causa da elevada pressão resultante desta concomitante redução, que não é compatível com os sistemas cromatográficos convencionais. Entretanto, o uso de partículas menores que $2 \mu \mathrm{m}$ se tornou possível recentemente, com o desenvolvimento da cromatografia líquida de ultra eficiência (CLUE).

\section{DESENVOLVIMENTO DA CROMATOGRAFIA LÍQUIDA AO LONGO DA SUA HISTÓRIA}

O desenvolvimento da CL, desde o seu início em 1950, quando se usavam colunas recheadas com partículas irregulares de 100-200 $\mu \mathrm{m}$ que alcançavam eficiências de apenas 200 pratos $/ 15 \mathrm{~cm}$, até hoje, está voltado para a tecnologia das partículas de recheio, associado à busca de melhor desempenho cromatográfico, melhor reprodutibilidade e análises mais rápidas sem perda de eficiência e de resolução. Como forma de elucidar a trajetória da evolução desta técnica, que atualmente emprega, com sucesso, partículas de FE menores que 2 $\mu \mathrm{m}$, os seus avanços estão detalhados no breve histórico ${ }^{1,2}$ descrito a seguir.

Ao redor dos anos 60, foram introduzidas as colunas recheadas com partículas peliculares rígidas de 40-50 $\mu \mathrm{m}$, mecanicamente resistentes e, dessa forma, próprias para serem usadas com pressão.

\footnotetext{
*e-mail: icsfj@iqm.unicamp.br
}

Estas partículas ofereciam maiores eficiências, 1000 pratos $/ 15 \mathrm{~cm}$, devido à taxa rápida de transferência de massa resultante da camada porosa fina das partículas peliculares, porém, apresentavam baixa área superficial e, conseqüentemente, baixa capacidade de amostra.

A transição para partículas porosas menores, com diâmetros em torno de $10 \mu \mathrm{m}$, ocorreu por volta dos anos 70, com a finalidade de contornar as desvantagens apresentadas pelas partículas de diâmetro maior e pelas peliculares. As colunas recheadas com partículas de $10 \mu \mathrm{m}$ apresentaram um ganho em eficiência, 6000 pratos $/ 15 \mathrm{~cm}$, quando comparadas às existentes até então. Entretanto, a reprodutibilidade do enchimento destas colunas com partículas menores e irregulares tornou-se um desafio, até serem desenvolvidas as partículas esféricas.

A partir dos anos 80, com a introdução das partículas esféricas, o desenvolvimento voltou-se basicamente para a redução do diâmetro das partículas de FE. As primeiras partículas esféricas desenvolvidas possuíam diâmetros de $5 \mu \mathrm{m}$ e apresentavam eficiência de 12000 pratos $/ 15 \mathrm{~cm}$ e, 10 anos mais tarde, foram introduzidas as partículas esféricas de 3-3,5 $\mu \mathrm{m}$ que alcançavam eficiências significativamente maiores que as anteriores, em torno de 22000 pratos $/ 15 \mathrm{~cm}$. Com isso, as análises tornaram-se 30-50\% mais rápidas e eficiências mais elevadas foram alcançadas, uma vez que estas partículas permitem enchimentos mais reprodutíveis e formam um leito cromatográfico mais homogêneo e compacto.

Em 1996, foram introduzidas as partículas esféricas não porosas de 1,5 $\mu \mathrm{m}$, com as quais foram obtidas colunas com eficiências de 30000 pratos $/ 15 \mathrm{~cm}$. Além da alta eficiência, estas partículas oferecem menor resistência à transferência de massa, facilitando o uso de uma faixa ampla de vazões de fase móvel, sem perda de desempenho cromatográfico e são mais resistentes e estáveis às altas pressões e temperaturas. Entretanto, devido à área superficial baixa, estas partículas resultam em menor capacidade de amostra que as partículas porosas e, por causa do tamanho pequeno, proporcionam tempos de retenção menores. Dessa forma, estes materiais são geralmente empregados para separação de macromoléculas como, por exemplo, as proteínas que têm difusão lenta na fase móvel.

Somente após 50 anos de desenvolvimento, surgiram as partículas esféricas porosas de $2,5 \mu \mathrm{m}$, com as quais são obtidas colunas com altas eficiências ( 25000 pratos $/ 15 \mathrm{~cm}$ ), os enchimentos são reprodutíveis e as análises são mais rápidas devido à concomitante diminuição dos tamanhos das colunas. Poucos anos mais tarde, surgiram as 
partículas esféricas porosas de 1,7 $\mu \mathrm{m}$ que, comparadas às partículas de 5 ou $3 \mu \mathrm{m}$, permitem melhores resoluções e altas eficiências, 30000 pratos $/ 15 \mathrm{~cm}$, porém, necessitam de uma instrumentação mais sofisticada para serem empregadas com o máximo de desempenho cromatográfico..$^{1-4}$

Os desenvolvimentos significativos alcançados na última década foram conseqüência do uso intenso da CLAE no mundo todo, como uma técnica de análise de rotina em laboratórios e, dessa forma, a busca por análises mais rápidas e com bom desempenho cromatográfico tornou-se evidente e necessária.

A alternativa mais simples, para diminuir o tempo de análise, seria o uso de colunas mais curtas associadas a vazões mais elevadas de fase móvel (FM). Com isso, as análises e os tempos de reequilíbrio da coluna seriam mais rápidos e as pressões seriam compatíveis com os sistemas convencionais de CLAE, devido ao menor comprimento da coluna. Esta alternativa foi aplicada, empregando-se partículas de FE de 3-3,5 $\mu \mathrm{m}$, mas mesmo assim as separações apresentavam menor desempenho cromatográfico e perda de resolução. ${ }^{4-6}$

Outra estratégia foi o uso das colunas monolíticas, ${ }^{1,7,8}$ que são um meio contínuo de separação, comumente em formato cilíndrico, altamente poroso e preparadas a partir de sílica ou de material polimérico. A estrutura é caracterizada por apresentar macroporos $(2 \mu \mathrm{m})$ e mesoporos (13 nm) que conferem aos monolitos eficiências similares às alcançadas com colunas recheadas com partículas de 3-3,5 $\mu \mathrm{m}$, porém, oferecem uma permeabilidade maior que os materiais particulados e, dessa forma, podem ser usadas vazões mais altas sem aumento da pressão no sistema cromatográfico e sem perda de eficiência, devido à rápida taxa de transferência de massa. Entretanto, o uso das colunas monolíticas está limitado às colunas comercialmente disponíveis, que são de um único fabricante, Merck, e apresentam diâmetros internos de 4,6 e 3,0 mm, não sendo tão compatíveis com o espectrômetro de massas (EM), além de consumirem maiores quantidades de solventes e possuírem estabilidade química limitada em uma estreita faixa de $\mathrm{pH}(2-8)$.

A busca por análises rápidas deu início à cromatografia líquida de alta temperatura (CLAT) $)^{1,5}$ que emprega colunas convencionais de CLAE, porém, as análises são realizadas em temperaturas maiores que $60{ }^{\circ} \mathrm{C}$. $\mathrm{O}$ uso de altas temperaturas diminui a viscosidade da fase móvel e, conseqüentemente, gera menores pressões no sistema cromatográfico, permitindo o uso de vazões de FM mais elevadas. Com isso são alcançadas maiores eficiências, maiores velocidades de transferência de massa e menores tempos de análise. Entretanto, esta técnica apresenta muitas desvantagens, entre elas, poucos materiais de recheio são estáveis a altas temperaturas, possibilidade de degradação dos compostos analisados e dificuldade de aquecimento e resfriamento da fase móvel, que restringem o seu uso em análises de rotina.

Recentemente, o uso de partículas menores que $2 \mu \mathrm{m}$, em sistemas cromatográficos com pressões convencionais, $40 \mathrm{MPa}(6000$ psi), tem diminuído bastante o tempo de análise, porém, o máximo desempenho cromatográfico tem sido limitado pela pressão. Para contornar o obstáculo da pressão e fazer uso das partículas menores que $2 \mu \mathrm{m}$, com o máximo de desempenho cromatográfico, tornando as análises até 20 vezes mais rápidas, sistemas capazes de trabalhar em altas pressões, $100 \mathrm{MPa}$ (15000 psi), foram desenvolvidos e esse avanço da cromatografia líquida foi denominado de cromatografia líquida de ultra eficiência (CLUE).

\section{INFLUÊNCIA DO TAMANHO DAS PARTÍCULAS EM CROMATOGRAFIA LÍQUIDA}

Como mostrado, o crescimento do emprego da cromatografia líquida deve-se à evolução dos materiais de recheio, que permitiram separações cada vez melhores. A base dessa evolução é governada pela equação de van Deemter, ${ }^{2,3,9-11}$ que descreve a relação entre eficiência, expressa pela altura de prato $(H, \mu \mathrm{m})$, velocidade linear da fase móvel $(\mu, \mathrm{mm} / \mathrm{s})$ e tamanho da partícula $\left(d_{p}\right)$ :

$H=A d_{p}+\frac{B D_{M}}{\mu}+\frac{C d_{p}^{2} \mu}{D_{M}}$

onde $D_{M}=$ coeficiente de difusão do analito e $A, B$ e $C$ são constantes.

$\mathrm{O}$ termo $A$ refere-se ao alargamento dos picos devido aos diferentes caminhos seguidos pelas moléculas do analito. Esse termo pode ser minimizado usando-se colunas de tamanho reduzido, com diâmetros internos menores, com enchimentos eficientes e partículas uniformes. $\mathrm{O}$ termo $B$ está relacionado à difusão longitudinal ou difusão do soluto na FM e este termo pode ser minimizado com o uso de altas velocidades lineares da FM. O termo $C$ descreve a transferência de massa do analito entre a FM e a FE.

O mínimo da curva de van Deemter representa a vazão ótima para se obter a eficiência máxima da coluna. Isto é um compromisso entre os termos $B$ e $C$. Os termos $A$ e $C$ são influenciados pelo tamanho das partículas. As partículas menores tendem a reduzir a altura do prato e, dessa forma, são obtidas colunas com um número de pratos maior, ou seja, mais eficientes. As partículas pequenas tendem a permitir trocas do soluto na FM e nos poros das partículas mais rapidamente devido a menor profundidade dos poros. Assim, o soluto gasta um tempo menor entre a FM e a FE, eluindo em picos estreitos.

Uma vantagem do uso de partículas menores que $2 \mu \mathrm{m}$ é que a eficiência da coluna pode ser mantida com a diminuição do seu comprimento. Uma coluna menor permite separações mais rápidas, uma vez que o tempo de separação é proporcional ao comprimento da coluna. Além disso, uma coluna menor, comparada a uma maior, empregando-se a mesma vazão de fase móvel, gasta uma menor quantidade de solvente, de amostra e de FE.

Na Figura 1, estão apresentadas as curvas de van Deemter, ${ }^{1,2,12-14}$ para partículas de $10,5,3 \mathrm{e} \leq 2 \mu \mathrm{m}$, que mostram que as partículas $\leq 2 \mu \mathrm{m}$ podem ser usadas para separações rápidas e ultra rápidas, uma vez que as eficiências são mantidas com uso de colunas curtas e com altas velocidades lineares de FM.

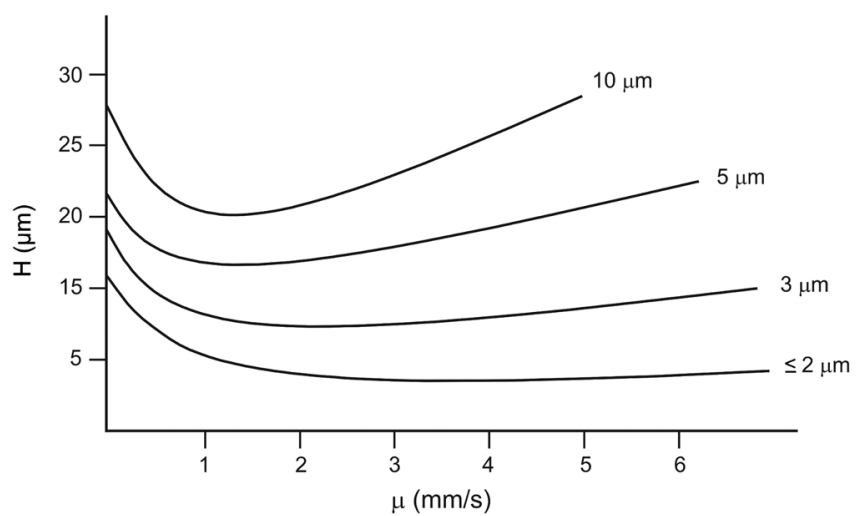

Figura 1. Curva de van Deemter para partículas de 10, 5, $3 e \leq 2 \mu m$. Adaptada da ref. 1

O uso de colunas recheadas com essas partículas $\leq 2 \mu \mathrm{m}$ requer a otimização de outros parâmetros experimentais.

A diminuição do tamanho da partícula provoca um aumento na pressão do sistema, conforme a Equação 2:6,15

$P=\phi \frac{L \eta \mu}{100 d_{p}^{2}}$ 
onde $P=$ pressão, $\phi=$ resistência à vazão, $L=$ comprimento da coluna $(\mathrm{mm}), \eta=$ viscosidade da FM $(\mathrm{mPa} / \mathrm{s}), \mu=$ velocidade linear $(\mathrm{mm} / \mathrm{s})$ e $d_{p}=$ tamanho da partícula $(\mu \mathrm{m})$.

Dessa forma, se o tamanho da partícula for reduzido pela metade, tem-se um aumento da pressão por um fator de 4. Porém, como o uso das partículas menores está associado ao emprego de colunas mais curtas, esse aumento na pressão passa a ser menor que 4 vezes, mas, mesmo assim, não é compatível com um sistema convencional de CLAE.

Outros fatores que precisam ser otimizados são o volume extra coluna e o tempo de residência dos gradientes, tempo que a FM leva para percorrer o sistema cromatográfico desde a saída do misturador até o topo da coluna, como forma de minimizar o alargamento do pico cromatográfico e obter a máxima eficiência da coluna.

\section{CROMATOGRAFIA LÍQUIDA ASSOCIADA AO USO DE ALTAS PRESSÕES}

A primeira citação relacionada ao uso de altas pressões, denominada cromatografia líquida de ultra alta eficiência (CLUAE), foi a de Bidlingmeyer et al., ${ }^{16} \mathrm{em} \mathrm{1969,} \mathrm{que} \mathrm{empregaram} \mathrm{partículas} \mathrm{menores}$ que $2 \mu \mathrm{m}$, em uma coluna longa e de diâmetro interno grande. Entretanto, os resultados obtidos foram irreprodutíveis devido a problemas no enchimento.

Os primeiros trabalhos relevantes usando pressões acima de 410 $\mathrm{MPa}$ (60000 psi) foram realizados por Jorgenson e colaboradores ${ }^{17} \mathrm{e}$ Lee e colaboradores, ${ }^{18}$ por volta de 1997 , nos quais usaram colunas capilares de sílica fundida $(30 \mu \mathrm{m}$ x $52 \mathrm{~cm})$ e partículas não porosas de 1-1,5 $\mu \mathrm{m}$ que resultaram em eficiências de 140000-190000 pratos. Esta técnica foi empregada na separação de benzodiazepínicos, de compostos farmacêuticos e de herbicidas. Entretanto, a dificuldade em adaptar um sistema de injeção apto a fazer a introdução da amostra em um sistema a alta pressão fez com que essa técnica ficasse restrita aos laboratórios acadêmicos que possuíssem sistemas de CLUAE de fabricação própria. ${ }^{10,12,19}$

O potencial de separação apresentado pelo CLUAE impulsionou as pesquisas para o desenvolvimento de um sistema adequado para trabalhar com altas pressões, porém, usando partículas de sílica porosas $(\leq 2 \mu \mathrm{m})$ e colunas analíticas de CLAE, o que deu origem à cromatografia líquida de ultra eficiência (CLUE).

\section{CROMATOGRAFIA LIQUIDA DE ULTRA EFICIÊNCIA (CLUE)}

A CLUE é o avanço mais recente das técnicas de separação, baseia-se nos mesmos princípios da cromatografia líquida de alta eficiência e utiliza fases estacionárias com partículas menores que $2 \mu \mathrm{m}$. O uso destas partículas juntamente com as altas velocidades lineares da FM aumentam a resolução e a detectabilidade e diminuem o tempo das análises. ${ }^{13,14,20-27}$ Para tornar isto possível, uma vez que a atual tecnologia de instrumentação (bombas, injetores e detectores) disponível para a CLAE não é projetada para trabalhar em altas pressões, um novo equipamento que pode operar em pressões acima de $100 \mathrm{MPa}$ foi introduzido e adaptado às necessidades atuais, em 2004 pela Waters Corporation. Este primeiro equipamento comercial capaz de operar em pressões acima de $100 \mathrm{MPa}$ (15000 psi) foi denominado pela Waters Corporation de Acquity ${ }^{T M}$ ultra performance liquid chromatography system (UPLC $\left.{ }^{\mathrm{TM}}\right) \cdot{ }^{14,20}$ Logo em seguida, outros dois fabricantes, a Jasco e a Agilent, lançaram seus instrumentos de CLUE, o Xtrem LC (X-LC), também com capacidade de trabalhar em pressões acima de $100 \mathrm{MPa}$ e o 1200 Series Rapid Resolution LC system capaz de trabalhar em pressões acima de $60 \mathrm{MPa}(9000$ psi), ${ }^{1,14,20}$ respectivamente.
As modificações requeridas em um sistema de CLUE são: capacidade de trabalhar a pressões muito altas (100 MPa), volumes internos muito menores (conexões, alça de amostragem, cela do detector, bombas), celas do detector sem dispersão e com alta taxa de aquisição, melhoramento no sistema de controle e de dados, colunas resistentes para trabalharem a altas pressões e com baixo volume morto, injetores com precisão na faixa de volumes pequenos.

\section{SISTEMA DE GERENCIAMENTO DE FASE MÓVEL}

Para se obter o máximo de aproveitamento das vantagens proporcionadas pelo tamanho reduzido das partículas e a fim de se alcançar elevada capacidade de separação em CLUE, uma instrumentação capaz de trabalhar em altas pressões é necessária. A pressão necessária, considerando a vazão ótima para alcançar o máximo de eficiência, empregando uma coluna de $15 \mathrm{~cm}$ recheada com partículas de FE de 1,7 $\mu \mathrm{m}$, fica ao redor de 15000 psi. Dessa forma, são requeridas bombas capazes de impulsionar a fase móvel de forma suave e reprodutível nestas altas pressões, para compensar a compressibilidade do solvente, e que sejam capazes de operar tanto no modo de eluição isocrático como por gradiente. ${ }^{6,12,14,20}$

Em um sistema de CLUE, devido à compressibilidade do solvente a altas pressões, o fluxo deve ser impulsionado de forma rápida e o sistema deve ter baixos volumes para se obter um bom desempenho.

Dessa forma, o equipamento de CLUE possui um sistema binário de solventes, com duas bombas individuais em série para propiciar gradientes a altas pressões. Nas saídas das bombas, o filtro e o misturador $(50 \mu \mathrm{L})$ estão acoplados em um mesmo dispositivo, para diminuir o volume do sistema e fornecer uma mistura adequada. O sistema tem um volume total de aproximadamente $150 \mu \mathrm{L}$. A vantagem de se fazer as misturas dos solventes a altas pressões é a obtenção de um baixo volume do sistema, uma vez que o tempo de residência da fase móvel é menor. ${ }^{14,20,28}$

\section{SISTEMA DE INJEÇÃO DA AMOSTRA}

As válvulas de injeção convencionais, tanto as manuais quanto as automáticas, não são projetadas e nem são rígidas o suficiente para operar em pressões extremas. Para proteger a entrada da coluna das flutuações resultantes do emprego das altas pressões, o processo de injeção deve ser feito em um curto intervalo de tempo e com um fluxo relativamente livre de pulso. Dessa forma, a injeção da amostra é realizada de forma automática e o amostrador possui controle de temperatura de $4-40{ }^{\circ} \mathrm{C} . .^{13,14}$

$\mathrm{O}$ volume de injeção de amostra deve ser reduzido para evitar o espalhamento da amostra e, consequientemente, o alargamento do pico cromatográfico. Para isto, os equipamentos de CLUE possuem uma faixa de volume de injeção de apenas $0,1-50 \mu \mathrm{L} .^{13,20}$

É necessário também um ciclo rápido para a injeção da amostra, para se obter o máximo de aproveitamento da velocidade oferecida pelo CLUE, uma vez que é requerida a separação de uma grande quantidade de compostos por unidade de tempo. Geralmente, nos sistemas de CLUE, o tempo de injeção da amostra sem a etapa da lavagem é de $25 \mathrm{~s}$, enquanto que com duas etapas de lavagem esse tempo passa para $60 \mathrm{~s}$. O processo de lavagem em duas etapas pode ser realizado utilizando dois solventes diferentes, geralmente, de polaridades distintas para a completa limpeza da agulha, como forma de reduzir o efeito de memória, ou seja, o arraste da amostra injetada em uma análise posterior e para aumentar a detectabilidade. ${ }^{1,13,20,28,29}$

Outra forma de anular o efeito de memória, além de reduzir os problemas de entupimento da agulha e diminuir a dispersão da amostra dentro da agulha de amostragem é usar agulhas do tipo needle-in-needle, ou seja, tem-se uma agulha dentro de outra agulha, 
sendo a agulha interna de PEEK (poliéter éter cetona) e a externa de aço inoxidável. ${ }^{13,20} \mathrm{~A}$ agulha de aço inoxidável é utilizada para perfurar o septo e a agulha de PEEK é empregada para sugar a amostra, uma vez que a adsorção da amostra no PEEK é menor que no aço inoxidável.

\section{COLUNAS E FASES ESTACIONÁRIAS}

O emprego de partículas $\leq 2 \mu \mathrm{m}$ como material de recheio das colunas cromatográficas aumenta a resistência ao fluxo da FM, ${ }^{6,14} \mathrm{de}$ modo que estas colunas devem operar em pressões mais altas. Além disso, as maiores eficiências destas colunas são alcançadas com o uso de altas velocidades lineares de FM, o que também contribui para o aumento da pressão no sistema cromatográfico, podendo atingir pressões maiores que $100 \mathrm{MPa}$.

Dessa forma, além de desenvolver FE resistentes, principalmente, ao uso de altas pressões, mudanças nos tubos das colunas e no processo de enchimento também foram necessárias.

Em termos de instrumentação, ${ }^{13,20,30}$ foi necessária a redução do diâmetro interno do tubo da coluna, de 3,0-4,6 mm das convencionalmente usadas para partículas de 3-5 $\mu \mathrm{m}$, para um diâmetro interno de 1,0-2,1 mm para as partículas $\leq 2 \mu \mathrm{m}$, a fim de minimizar o efeito do aquecimento dos solventes da fase móvel, que ocorre quando são usadas altas pressões. Este aquecimento pode provocar um gradiente de temperatura ao longo da corrida, gerando uma vazão de FM não uniforme, o que pode prejudicar as separações tornando-as irreprodutíveis.

Foi necessário também o desenvolvimento de novos filtros de coluna, com porosidade menor que $2 \mu \mathrm{m}$ para reter as partículas das $\mathrm{FE}$, capazes de resistirem às altas pressões e a um grande número de injeções, sem apresentarem problemas de entupimento, uma vez que a porosidade é extremamente reduzida. ${ }^{1,14}$

Para tornar o sistema de enchimento próprio para o recheio de colunas curtas e com FE da ordem de $2 \mu \mathrm{m}$ foi necessária a ampliação da faixa de pressão máxima que pode ser aplicada, que passou de 100 MPa (15000 psi) dos sistemas de enchimento comumente utilizados para colunas com partículas de 3-5 $\mu \mathrm{m}$ para $134 \mathrm{MPa}$ (20000 psi) para as partículas $\leq 2 \mu \mathrm{m}$. Esta etapa é considerada crítica, devido à dificuldade encontrada na reprodutibilidade do enchimento das colunas com partículas com este tamanho. ${ }^{20,30}$

Em relação às $\mathrm{FE}$ utilizadas em cromatografia líquida de forma geral, muitos avanços já aconteceram, como descrito ao longo do texto, porém as FE próprias para serem empregadas em CLUE ainda são alvo de desenvolvimento, devido ao nível de excelência exigido por estes sistemas.

As partículas de sílica nua, existentes até então, não possuíam a resistência mecânica ou a eficiência necessária que as separações em CLUE demandam. As partículas a serem empregadas em CLUE devem apresentar, como um dos primeiros requisitos, maior resistência física ou mecânica, porém, esta característica não pode estar associada à diminuição de capacidade de amostra, como é o caso das partículas não porosas, menores que $2 \mu \mathrm{m}$.

É recomendável também, que as novas partículas sejam estáveis a uma grande faixa de $\mathrm{pH}$ e que as interações indesejáveis com os compostos analisados sejam minimizadas. ${ }^{14,20,30}$ Estas características, porém, não são somente desejáveis quando se trata de CLUE e sim em qualquer modalidade da cromatografia líquida.

\section{TIPOS DE COLUNAS DE CLUE}

Atualmente, vários fabricantes s,5,6,30 $^{2}$ êm desenvolvido colunas com dimensões adequadas para CLUE, conforme mostra a Tabela 1 , porém apenas as colunas do tipo Acquity ${ }^{\mathrm{TM}}$ UPLC BEH, fabricadas pela Waters, preenchem todos os requisitos necessários para trabalhar na condição de máximo desempenho da CLUE. O diferencial das colunas Acquity ${ }^{\mathrm{TM}}$ UPLC BEH das demais é o emprego de partículas híbridas (orgânicas e inorgânicas) que são mais resistentes mecanicamente que as partículas de sílica pura. ${ }^{2}$

Tabela 1. Fabricantes, denominação e tamanho das partículas das colunas cromatográficas desenvolvidas para CLUE

\begin{tabular}{lcc}
\hline Fabricante & Coluna & $\begin{array}{c}\text { Tamanho da } \\
\text { partícula }(\mu \mathrm{m})\end{array}$ \\
\hline Waters & Acquity ${ }^{\mathrm{TM}}$ UPLC BEH & 1,7 \\
Agilent & Zorbax & 1,8 \\
Thermo Electron & Hypersil GOLD & 1,9 \\
Bischoff & ProntoPEARL & 1,8 \\
Altech & Platinum HPLC & 1,5 \\
Macherey Nagel & Nucleodur & 1,8 \\
Shimadzu & Pathfinder & 1,5 \\
Interchim & TSKgel Super-ODS & 2,0 \\
Sepax & GP Series & 2,0 \\
YMC & YMC ultra-fast & 2,0 \\
\hline
\end{tabular}

A tecnologia envolvida na obtenção dessas partículas será descrita e discutida, tendo como base as colunas do tipo Zorbax e Acquity ${ }^{\mathrm{TM}}$ UPLC BEH, com o objetivo de mostrar os desenvolvimentos mais recentes no que diz respeito às fases estacionárias para CLUE.

\section{Colunas Zorbax}

O desenvolvimento das FE das colunas Zorbax com tamanho de partículas reduzido baseou-se na busca de melhor estabilidade química em condições mais agressivas de FM, uma vez que a faixa de $\mathrm{pH}$ da sílica está limitada entre 2 e 8 , de melhor estabilidade física e de maior tempo de vida útil.

As colunas Zorbax Eclipse Extra Densely Bonded (XDB) consistem de uma FE de 1,8 $\mu$ m do tipo C18, C8 ou fenil que é duplamente capeada (Figura $2 \mathrm{~A}$ ), o que reduz o número de silanóis livres para um valor quase nulo, podendo ser usadas em uma faixa de $\mathrm{pH}$ de 2-9. Essas FE são convenientes para a separação de compostos neutros, básicos e ácidos. ${ }^{2,31} \mathrm{O}$ processo de capeamento consiste em uma reação de silanização com um reagente organossilano de tamanho reduzido, após a qual se tem uma redução do número de silanóis residuais.

Outro tipo de coluna disponível é a Zorbax StableBond (SB) que é preparada a partir de silanos monofuncionais com cadeias $\mathrm{C} 3$, $\mathrm{C} 8, \mathrm{C} 18$, fenil e $\mathrm{CN}$ e de grupos volumosos que protegem estericamente as ligações siloxano (Figura 2 B). Para as FE do tipo C18 os grupos protetores podem ser diisobutil ou diisopropil, enquanto que para as FE dos tipos C8, C3, fenil e CN são empregados os grupos diisopropil. Estas FE estericamente protegidas fornecem maior estabilidade e aumentam o tempo de vida e a reprodutibilidade destas FE quando são usadas FM ácidas, uma vez que elas resistem a uma faixa de $\mathrm{pH}$ de 1-6 e são compatíveis com FM com elevada quantidade de água. ${ }^{2,31}$

\section{Colunas Acquity ${ }^{\mathrm{TM}}$}

$\mathrm{O}$ desenvolvimento das colunas Acquity ${ }^{\mathrm{TM}}$ foi direcionado às peculiaridades impostas pela CLUE, como a preparação de partículas de FE especiais. Para atender este requisito, foram desenvolvidas as partículas híbridas, produzidas pela reação de bis(trietoxissililetano) e 
(A)

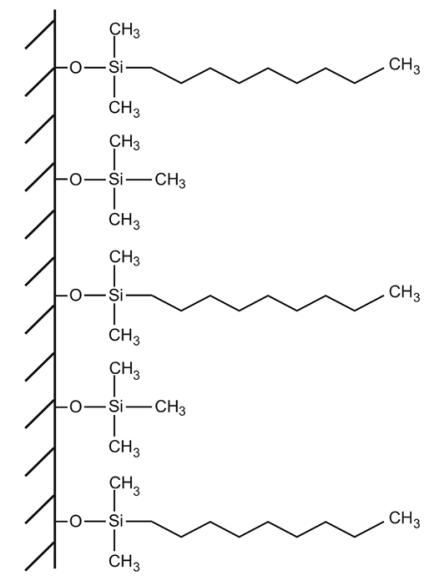

(B)

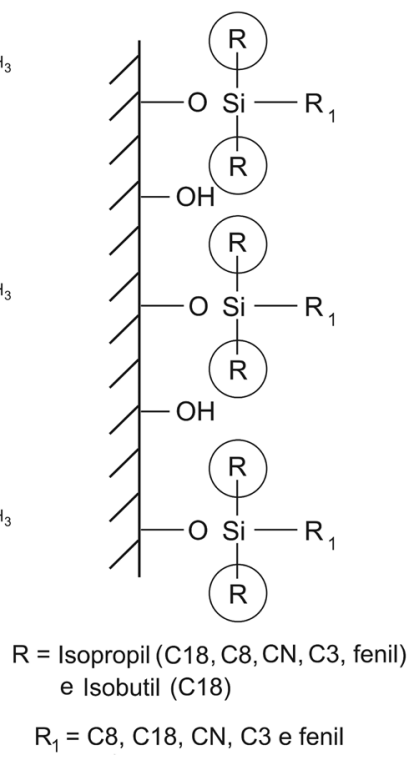

Figura 2. Estruturas das fases estacionárias: (A) Zorbax Eclipse eXtra Densely Bonded C8 e (B) Zorbax StableBond. Adaptada da ref. 31

tetraetoxissilano, que dá origem a uma partícula de sílica com pontes de etano inseridas na sua estrutura (polietoxissilano), denominada de sílica híbrida de segunda geração (Figura 3). Estas partículas são muito diferentes das partículas tradicionais de sílica, porque as modificações têm resultado em adição de carbono (grupos etil) nas ligações siloxano, semelhante ao que ocorre no processo de capeamento.

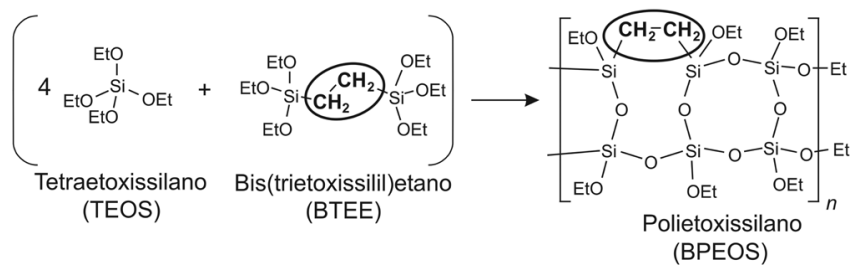

Figura 3. Equação da reação química de síntese da sílica híbrida de segunda geração. Adaptada da ref. 30

A combinação de material orgânico e inorgânico gera partículas com diâmetros de 1,7 m que possuem maior estabilidade mecânica, uma vez que as ligações silício-carbono são similares às ligações silício-oxigênio; são resistentes em uma faixa extensa de $\mathrm{pH}(1-12)$ e esta maior estabilidade química é conferida pelas pontes de carbono que estão inseridas na superfície da sílica; são estáveis em temperaturas de até $60^{\circ} \mathrm{C}$ e fornecem uma boa simetria de pico, devido à quantidade reduzida de silanóis residuais. ${ }^{1,2,5,69,12-14,30}$

Cabe ressaltar ainda que estas partículas conferem um aumento na resolução dos picos cromatográficos, devido às altas eficiências fornecidas por estas colunas. Além disso, as partículas híbridas de $1,7 \mu \mathrm{m}^{14,20,30}$ são obtidas em uma distribuição estreita de tamanho de partículas, que pode ser visualizada na Figura 4, o que além de facilitar o processo de enchimento das colunas, gera um leito cromatográfico mais homogêneo e eficiente.

Encontram-se disponíveis comercialmente 4 colunas Acquity ${ }^{\mathrm{TM}}$ UPLC BEH, ${ }^{2,30}$ com diferentes características, que empregam partículas híbridas de segunda geração de 1,7 $\mu$ m: C18 e C8 - são recheadas com FE contendo cadeias alquil ligadas à superfície da sílica, de forma a oferecer melhor estabilidade hidrolítica. As fases estacionárias com

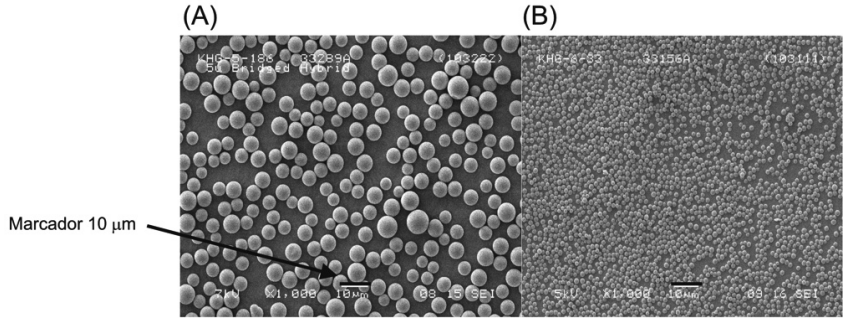

Figura 4. Microscopia das partículas de sílica: (A) pura de 5 um e (B) híbrida de segunda geração de 1,7 $\mu$ m. Adaptada da Waters Corporation

cadeias de $\mathrm{C} 8$ são menos hidrofóbicas e, conseqüentemente, oferecem menor retenção. Ambas as fases estacionárias encontram-se capeadas; Shield RP18 - é a denominação dada às colunas recheadas com FE que possuem grupos carbamatos embutidos, que conferem uma retenção diferenciada para os compostos doadores de hidrogênio. Estas FE são capeadas com trimetilsilano; C6 Fenil - são recheadas com FE do tipo fenil que conferem uma seletividade específica para compostos aromáticos ou outros compostos com elétrons $\pi$. Estas também são FE capeadas.

Poucos estudos foram realizados para avaliar a estabilidade destas novas colunas para CLUE. As colunas Acquity ${ }^{\mathrm{TM}}$ foram avaliadas por King et $a l .{ }^{32} \mathrm{e}$ por Grumbach et $a l .,{ }^{30}$ quanto à resistência mecânica a altas pressões e à estabilidade química, usando uma FM tamponada com metilpirrolidina a pH 11,3, respectivamente. Os resultados obtidos mostraram, respectivamente, que a estabilidade dessas colunas foi de 4000 e de 1000 injeções. Kaufmann e Butcher ${ }^{33}$ verificaram o aparecimento de picos duplos, com o uso de pressões entre 73-82 MPa (11000-13000 psi), e associaram o problema ao entupimento dos filtros, ocasionado durante o enchimento das colunas.

Dessa forma, é difícil avaliar a estabilidade de uma coluna, uma vez que são muitos os fatores envolvidos, como, solventes e $\mathrm{pH}$ da FM, pressões utilizadas, complexidade da amostra injetada, temperatura de análise, entre outros. Porém, todos os trabalhos publicados mostram que as colunas apresentam boa estabilidade nas condições utilizadas.

\section{SISTEMA DE DETECÇÃO}

Com o uso das partículas $\leq 2 \mu \mathrm{m}$ são obtidos picos cromatográficos com largura a meia altura menores que $1 \mathrm{~s}$, exigindo, dessa maneira, mudanças significativas nos detectores. De forma geral, para manter a exatidão e a reprodutibilidade no processo de aquisição dos dados, tanto os detectores ópticos, UV-VIS e arranjo de diodos (DAD), como os espectrômetros de massas precisam ser capazes de operar com altas taxas de amostragem, para adquirir um número de pontos suficientes para a construção do pico cromatográfico. Teoricamente, a detectabilidade de um sistema de CLUE, comparada com a de um de CLAE, é cerca de 2-3 vezes maior, dependendo do tipo de detector usado., ${ }^{1,12,14}$

Mudanças mais significativas ocorreram nos detectores ópticos convencionais (UV e DAD) que são projetados para equipamentos de CLAE, nos quais são injetadas maiores quantidades de amostra e o tempo de análise é mais longo, quando comparado com o de CLUE.

Assim, esses detectores necessitam de diminuição do volume da cela do detector e um longo caminho óptico, para manter a concentração e o sinal e uma baixa dispersão da amostra, a fim de se obter alta detectabilidade. As dimensões de volume da cela e o comprimento do caminho óptico, desses detectores, são de aproximadamente 500 $\mathrm{nL}$ e $10 \mathrm{~mm}$, respectivamente. ${ }^{13,14,20}$ 
A constante de tempo do detector deve ser suficientemente rápida ( $\leq 100 \mathrm{~ms}$ ) devido às estreitas larguras de base dos picos (apenas poucos segundos) e às altas taxas de amostragem (>20 Hz) e de aquisição dos dados (20-40 pontos/s) que são necessárias para adquirir dados suficientes em curto espaço de tempo. . $^{13,14,20}$

\section{CARACTERIZAÇÃO DO DESEMPENHO CROMATOGRÁFICO DAS COLUNAS DE CLUE}

A cromatografia líquida de ultra eficiência, por ser uma técnica bastante recente, ainda está em processo de avaliação por pesquisadores da área e também pelos usuários da cromatografia líquida em diversos tipos de análises, visando estabelecer as vantagens que esta nova modalidade oferece em relação à cromatografia líquida de alta eficiência.

Sob este ponto de vista, alguns trabalhos foram desenvolvidos, como o de Nováková et al. ${ }^{12}$ que desenvolveram um método para analisar os compostos de uma formulação farmacêutica de diclofenaco. Estas análises foram realizadas por CLUE, empregando as colunas Acquity ${ }^{\mathrm{TM}}$ BEH C18 $(50 \times 2,1 \mathrm{~mm}, 1,7 \mu \mathrm{m})$ e $(100 \times 2,1$ $\mathrm{mm}, 1,7 \mu \mathrm{m})$ e por CLAE, empregando as colunas Zorbax Eclipse XDB-C18 (75 x 4,6 mm, 3,5 $\mu \mathrm{m})$, Zorbax Eclipse SB-C18 (50 x 4,6 mm, 1,8 $\mu \mathrm{m})$, Purospher RP18e (125 x 4,0 mm, 5,0 $\mu \mathrm{m})$ e uma coluna monolítica Chromolith Performance RP-18e (100 x 4,6 mm). Do ponto de vista de reprodutibilidade do tempo de retenção e da área dos picos cromatográficos, as colunas Zorbax com partículas de 1,8 e $3,5 \mu \mathrm{m}$ apresentaram melhores resultados que as colunas Acquity ${ }^{\mathrm{TM}}$ e isto pode estar relacionado à baixa precisão, devida ao uso parcial da agulha de injeção. Em relação à simetria de pico, resolução e eficiência, as colunas Acquity ${ }^{\mathrm{TM}}$ apresentaram resultados substancialmente melhores que os obtidos para as demais colunas. Os melhores resultados de tempo de análise e de consumo de solvente foram obtidos com as colunas Acquity ${ }^{\mathrm{TM}}$. Com a coluna Acquity ${ }^{\mathrm{TM}}$ $(50 \times 2,1 \mathrm{~mm}, 1,7 \mu \mathrm{m})$ o tempo de análise foi de 2,4 min e o consumo de FM, metanol:água acidificada com ácido fosfórico a pH 2,5 (65:35 v/v) a uma vazão de $0,45 \mathrm{~mL} / \mathrm{min}$, foi de $1,08 \mathrm{~mL}$, enquanto que com a coluna Purospher $(125 \times$ 4,0 mm, 5,0 $\mu \mathrm{m})$ o tempo de análise foi de $13 \mathrm{~min}$ e o consumo de FM (vazão de $0,7 \mathrm{~mL} / \mathrm{min}$ ) foi de 11,9 $\mathrm{mL}$. Com as colunas Zorbax de 1,8 $\mu \mathrm{m}$ o tempo de análise foi similar aos obtidos com as colunas Acquity ${ }^{\mathrm{TM}}$ (3,5 min), e com a coluna monolítica foi obtido o menor tempo de análise (1,8 min), porém todas estas colunas apresentaram um maior consumo de solvente em relação às colunas Acquity ${ }^{\mathrm{TM}}$, por causa das maiores vazões de FM requeridas por estas colunas.

Outra avaliação interessante apresentada neste trabalho refere-se ao uso das colunas convencionais de CLAE em CLUE. Para isso, as colunas Zorbax foram conectadas ao sistema de CLUE e as mesmas análises foram realizadas. Os resultados mostraram que esta operação pode ser facilmente realizada, porém não apresenta vantagem significativa, devido à necessidade do uso de vazões de FM menores que aquelas empregadas no sistema de CLAE. Dessa forma, o uso de colunas convencionais no sistema de CLUE não gera análises mais rápidas, porém foi verificada uma melhora na simetria do pico cromatográfico.

Nguyen et al. ${ }^{6}$ compararam colunas recheadas com partículas $\leq 2 \mu \mathrm{m}$, usando um sistema de CLUE, com colunas convencionais recheadas com partículas de 3,5 e $5 \mu \mathrm{m}$, usando um sistema de CLAE, na análise de uma mistura de 4 alquilparabenos. Os resultados destas comparações mostraram que: (a) as colunas recheadas com partículas de $1,7 \mu \mathrm{m}$ apresentaram eficiências três vezes maiores e as análises foram oito vezes mais rápidas que as colunas recheadas com partículas de $5 \mu \mathrm{m}$. Isto pode ser verificado visualizando a Figura 5, porém é importante observar que a escala do eixo das abscissas (tempo de análise) foi expandida para a coluna Acquity ${ }^{\mathrm{TM}}$; (b) o número de pratos por unidade de tempo (pratos/s), que é um parâmetro que descreve o compromisso entre alta eficiência e análises rápidas, foi maior que 1000 pratos/s, que é um valor muito bom, uma vez que são sugeridos valores entre 400-1000 pratos/s quando se emprega condições de CLUE e em torno de 100 pratos/s quando se emprega cromatografia líquida convencional; (c) os valores de resistência ao fluxo de fase móvel apresentados pelas colunas recheadas com partículas de 1,7 $\mu \mathrm{m}$ foram maiores que os valores apresentados pelas colunas recheadas com partículas de 1,9 $\mu \mathrm{m}$, sugerindo, dessa forma, que as colunas recheadas com partículas de $1,7 \mu \mathrm{m}$ possuem uma maior compactação do leito cromatográfico e uma distribuição mais estreita de tamanho de partículas, uma vez que a permeabilidade e a resistência ao fluxo não variam com a natureza das partículas do leito cromatográfico, mas sim com os "espaços" entre as partículas e com a compactação do mesmo; (d) as colunas recheadas com as partículas $\leq 2 \mu \mathrm{m}$ apresentaram boa estabilidade durante algumas centenas de injeções, enquanto que as colunas recheadas com as partículas de $5 \mu \mathrm{m}$ se mostraram estáveis a um número bem maior de injeções (500-1000). Esta diferença pode estar relacionada com a nova geometria das colunas de CLUE, as altas pressões de enchimento e a pequena porosidade dos filtros, que podem entupir após um grande número de injeções.

Com o mesmo intuito de comparação descrito acima, Villiers et al. ${ }^{9}$ realizaram um trabalho empregando colunas recheadas com partículas de 1,7 $\mu \mathrm{m}$ em um sistema de CLUE e colunas recheadas com partículas de 3,5 e $5 \mu \mathrm{m}$ em um sistema de CLAE, na análise de uma mistura de uracila, cafeína, piridina, anilina, fenol, acetofenona e benzeno, geralmente usada para a avaliação do desempenho cromatográfico de colunas convencionais de CLAE. Os resultados obtidos confirmam os conceitos introduzidos neste texto: (a) a velo-

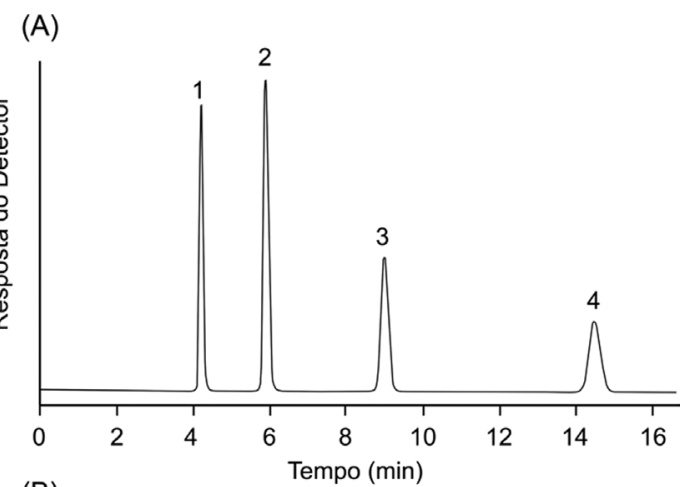

(B)

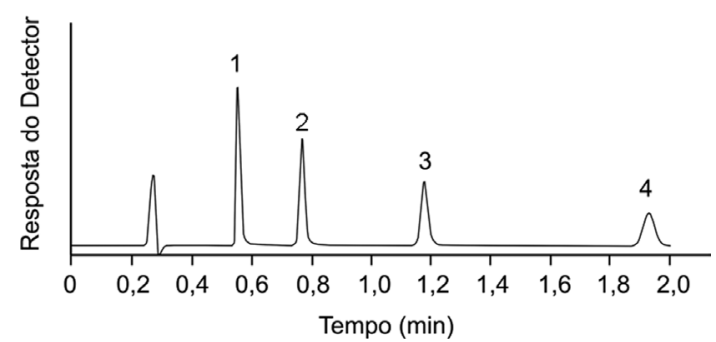

Figura 5. Cromatogramas obtidos na separação dos alquilparabenos (1 metilparabeno, 2 - etilparabeno, 3 - propilparabeno, 4 - butilparabeno). (A) Coluna XTerra RP18 $(4,6 \times 150 \mathrm{~mm}, 5 \mu \mathrm{m})$. Condições Cromatográficas: FM: acetonitrila:água (40:60 v/v), vazão da FM: $900 \mu \mathrm{L} / \mathrm{min}$, temperatura: $30{ }^{\circ} \mathrm{C}$, volume de injeção: $10 \mu \mathrm{L}$ e detecção por arranjo de diodos a 254 nm. (B) Coluna Acquity ${ }^{T M}$ BEH C18, (2,1 x $\left.50 \mathrm{~mm}, 1,7 \mu \mathrm{m}\right)$. Condições Cromatográficas: FM: acetonitrila:água (40:60 v/v), vazão da FM: $520 \mu \mathrm{L} /$ min, temperatura: $30^{\circ} \mathrm{C}$, volume de injeção: $1 \mu \mathrm{L}$ e detecção por arranjo de diodos a $254 \mathrm{~nm}$. Adaptada da ref. 6 
cidade linear ótima e a altura de prato obtida para a coluna recheada com partículas de $1,7 \mu \mathrm{m}$ foram respectivamente de $0,37 \mathrm{~cm} / \mathrm{s}$ e 4,4 $\mu \mathrm{m}$, enquanto que os valores para a coluna recheada com partículas de $5 \mu \mathrm{m}$ foram de $0,17 \mathrm{~cm} / \mathrm{s}$ e $9,4 \mu \mathrm{m}$, comprovando experimentalmente que as partículas de diâmetros menores fornecem maiores eficiências com o aumento das velocidades lineares de fase móvel; (b) a combinação da vazão ótima com colunas mais curtas recheadas com partículas de $1,7 \mu \mathrm{m}$ proporcionou uma diminuição no tempo de análise de 4,3 e 3,5 vezes, quando comparadas com as colunas recheadas com partículas de 5 e $3,5 \mu \mathrm{m}$, respectivamente, sem diminuir a eficiência das colunas; (c) a avaliação da formação de gradiente de temperatura com o emprego de altas pressões foi realizada analisando a perda de eficiência de colunas isoladas em espuma de poliestireno, simulando um meio adiabático, e de colunas não isoladas, usando as vazões ótimas e as vazões que resultassem na pressão limite do CLUE. Os resultados mostraram que existe uma perda na eficiência de aproximadamente $10 \%$ somente quando a coluna não está isolada e com a aplicação de vazões mais altas, mostrando que o gradiente de temperatura não tem muita influência quando se utiliza a vazão ótima de análise.

\section{TRANSFERÊNCIA DE MÉTODOS DE CLAE PARA CLUE}

A possibilidade de transferência de um método já desenvolvido e protocolado empregando um sistema de CLAE para um de CLUE é um aspecto muito importante a ser considerado, pois, muitas vezes, tem-se constituído no fator limitante para difusão e uso da CLUE. Segundo os estudos de Veuthey e colaboradores, ${ }^{24,26}$ a transferência de um método de CLAE já desenvolvido para CLUE é totalmente passível, desde que determinados fatores sejam considerados. Estes fatores dependem do modo de eluição empregado no método de separação já desenvolvido.

Na transferência de um método de separação no modo de eluição isocrática, ${ }^{24}$ o principal fator que influencia é o volume extra coluna (do injetor, da cela do detector e das tubulações), que provoca alargamento do pico cromatográfico quando não existe compatibilidade entre o volume extra coluna do equipamento com o diâmetro da coluna, ou seja, para se obter uma boa separação a razão entre o volume extra coluna e o volume total (extra coluna e da coluna) deve ser menor que $10 \%$, tanto em CLAE quanto em CLUE. Além disso, para a transferência do método ser eficiente a fase estacionária empregada deve ser similar à do método já existente. Os parâmetros a serem otimizados são o volume de injeção e a vazão da fase móvel, que podem ser calculados pelas Equações 3 e 4, respectivamente, ou pelo programa desenvolvido e disponibilizado pelos mesmos autores, denominado de HPLC calculator v.2.0. ${ }^{34}$

$V_{i n j 2}=V_{i n j_{1}} \times \frac{d_{c_{2}}^{2}}{d_{c_{1}}^{2}} \times \frac{L_{2}}{L_{1}}$

onde, $V_{i n j 2}=$ volume de injeção a ser determinado, $V_{i n j l}=$ volume de injeção do método desenvolvido, $d_{c 2}=$ diâmetro interno da coluna a ser empregada, $d_{c l}=$ diâmetro interno da coluna empregada no método desenvolvido, $L_{2}=$ comprimento da coluna a ser empregada e $L_{1}=$ comprimento da coluna empregada no método desenvolvido.

$F_{2}=F_{1} \times \frac{d_{c_{2}}^{2}}{d_{c_{1}}^{2}} \times \frac{d_{p_{1}}}{d_{p_{2}}}$

onde, $F_{2}=$ vazão da fase móvel a ser determinada, $F_{1}=$ vazão da fase móvel do método desenvolvido, $d_{p l}=$ diâmetro da partícula de FE empregada no método desenvolvido e $d_{p 2}=$ diâmetro da partícula de $\mathrm{FE}$ a ser empregada.
$\mathrm{Na}$ transferência de um método de separação no qual foi empregada eluição por gradiente, ${ }^{26} \mathrm{o}$ fator principal a ser considerado é o tempo de residência da FM que resulta em variações nos tempos de retenção, comprometendo a resolução, sobretudo dos primeiros compostos eluídos, quando não existe compatibilidade entre o tempo de residência da FM com o diâmetro da coluna. Dessa forma, a transferência só é válida quando o tempo de residência da FM é desprezível, ou seja, quando a razão entre o tempo de residência da FM e o tempo de retardamento da FM é constante. Além disso, para a transferência do método ser eficiente a fase estacionária empregada deve ser similar à usada no método já existente e as composições inicial e final do gradiente devem permanecer constantes. Os parâmetros a serem otimizados são o volume de injeção, a vazão da fase móvel, o tempo da etapa isocrática do gradiente e o tempo do gradiente que podem ser calculados pelas Equações 3, 4, 5 e 6, respectivamente, ou pelo HPLC calculator v.2.0. ${ }^{34}$

$t_{i s o_{2}}=t_{i s o_{1}} \times \frac{F_{1}}{F_{2}} \times \frac{V_{m_{2}}}{V_{m_{1}}}$

onde, $t=$ tempo, $V_{m 2}=$ volume de retardamento da FM a ser empregado e $V_{m l}=$ volume de retardamento da FM empregado no método desenvolvido.

$t_{\text {grad }_{2}}=\frac{\left(\% B_{\text {final }_{1}}-\% B_{\text {inicial }_{1}}\right)}{\text { slope }_{2}}$

onde, $\% B=$ - porcentagem de solvente orgânico e

slope $_{2}=$ slope $_{1} \times \frac{V_{m_{1}}}{V_{m_{2}}} \times \frac{F_{2}}{F_{1}}$

O sucesso da transferência dos métodos, nos dois modos de eluição, pode ser facilmente visualizado nos dois exemplos a seguir. $\mathrm{O}$ primeiro é referente à transferência de um método desenvolvido empregando CLAE para a separação de uma mistura de rapidocaína, ${ }^{24}$ usando eluição isocrática, para CLUE (Figura 6). Comparando-se os cromatogramas apresentados na Figura 6, pode-se perceber que a transferência do método é plausível de ser realizada, e, além disso, o tempo de análise reduziu para $40 \mathrm{~s}$, a resolução permaneceu constante e a eficiência aumentou em torno de $10 \%$. O segundo exemplo refere-se à transferência de um método desenvolvido empregando CLAE para a separação de uma formulação farmacêutica, ${ }^{26}$ no modo de eluição gradiente, para CLUE. Os cromatogramas apresentados na Figura 7 mostram que a transferência de um método por eluição gradiente também pode ser realizada e, além disso, o tempo de análise reduziu por um fator de 10 , a detectabilidade não foi afetada, a resolução diminuiu somente 10\% para o par 3-4 (que são os compostos mais afetados pelo tempo de residência da fase móvel) e o tempo de reequilíbrio da coluna passou de 20 para $2 \mathrm{~min}$.

\section{APLICAÇÕES DA CLUE}

As primeiras aplicações empregando um CLUE foram realizadas com um sistema acoplado a um EM com analisador de tempo de vôo em análises de metabonomas e genomas. ${ }^{13}$ Atualmente, muitos trabalhos já foram realizados, empregando CLUE com os detectores ópticos (UV e DAD) e acoplado à espectrometria de massas, nas mais diversas áreas. Vários trabalhos envolvem a determinação de fármacos, ${ }^{21,25,27,35-41}$ por exemplo, Dongre et al. ${ }^{21}$ fizeram a validação de um método para a determinação de primaquina e suas impurezas usando colunas recheadas com partículas de 1,7 $\mu \mathrm{m}$, em um CLUEUV, e colunas recheadas com partículas de $5 \mu \mathrm{m}$, em um CLAE-UV. 
(A)

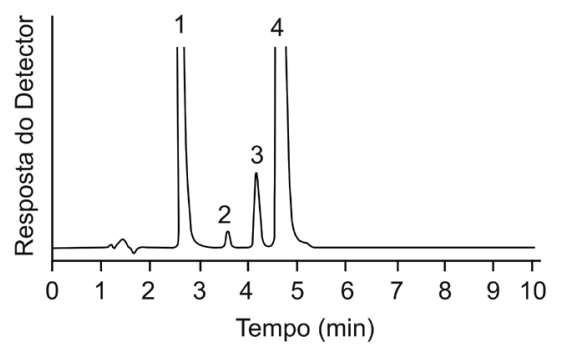

(B)

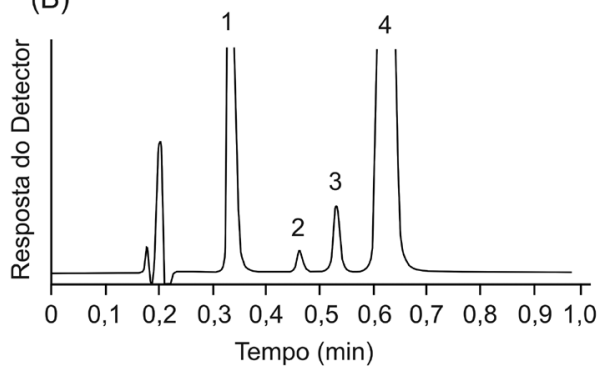

Figura 6. Cromatogramas ilustrativos da transferência de um método de separação no modo de eluição isocrática, desenvolvido para CLAE (A) e transferido para CLUE $(B)$ para a separação de uma mistura de rapidocaína (1-metilparabeno, 2-2,6-dimetilanilina, 3-propilparabeno, 4-lidocaína). (A) Condições Cromatográficas: Coluna XTerra RP18 (150 x 4,6 mm, $5 \mu \mathrm{m})$, FM: acetonitrila:tampão fosfato pH 7,2 (50:50 v/v), vazão da FM: $1000 \mu \mathrm{L}$ min, volume de injeção: $20 \mu \mathrm{L}$, temperatura: $30^{\circ} \mathrm{C}$ e detecção UV a $230 \mathrm{~nm}$. (B) Condições Cromatográficas: Coluna Acquity ${ }^{T M}$ BEH C18 $(50 \times 2,1 \mathrm{~mm}$, 1,7 $\mu \mathrm{m}), F M$ : acetonitrila:tampão fosfato $\mathrm{pH}$ 7,2 (50:50 v/v), vazão da FM: $610 \mu \mathrm{L} / \mathrm{min}$, volume de injeção: $1,4 \mu \mathrm{L}$, temperatura: $30^{\circ} \mathrm{C}$ e detecção $U V$ a $230 \mathrm{~nm}$. Adaptada da ref. 24

Os resultados obtidos empregando o primeiro sistema em relação ao segundo foram superiores em três pontos principais: tempo de análise (14 vezes mais rápido), eficiência e detectabilidade ( 2 vezes maior). A detectabilidade foi medida pelos limites de detecção (LD) e de quantificação (LQ), sendo que os LQ estão apresentados na Figura 8 , na qual se pode avaliar, com clareza, o aumento da detectabilidade com uso de colunas recheadas com partículas $\leq 2 \mu \mathrm{m}$ e CLUE, uma vez que as duas técnicas empregaram o mesmo detector.

A determinação de agrotóxicos ${ }^{4,42,43}$ também vem sendo explorada com o emprego de CLUE, e algumas pesquisas já foram desenvolvidas, como a de Kovalczuk et al.,${ }^{4}$ que determinaram 16 agrotóxicos em maçãs por CLUE-EM-EM e CLAE-EM-EM. As duas técnicas possibilitaram a determinação dos compostos, porém o método desenvolvido para o CLUE-EM-EM consumiu uma menor quantidade de solvente, devido ao menor tempo de análise e forneceu limites de quantificação menores que os obtidos por CLAE-EM-EM, para a maioria dos compostos. Os LQ empregando CLUE-EM-EM ficaram entre 0,5-8,0 $\mu \mathrm{g} / \mathrm{kg}$ e empregando CLAE-EM-EM ficaram entre 2,0$8,0 \mu \mathrm{g} / \mathrm{kg}$, dependendo do composto. Este aumento de detectabilidade proporcionado pelo uso do CLUE é importante, uma vez que os limites máximos de resíduos de agrotóxicos exigidos pelas agências reguladoras têm sido cada vez menores.

Outra aplicação interessante realizada por Bendahl et al. ${ }^{44}$ foi a determinação de conservantes em cosméticos à base de bromo (bronopol, bronidox e metildibromo glutaronitrila) por CLUE-PAI-EM, ou seja, CLUE acoplado a um plasma de argônio com acoplamento indutivo (PAI) em série a um EM, que se mostrou uma técnica analítica muito atrativa, por combinar uma técnica de separação de alta eficiência com um detector muito sensível e específico a um elemento. Os resultados mostraram que o emprego de altas velocidades lineares
(A)
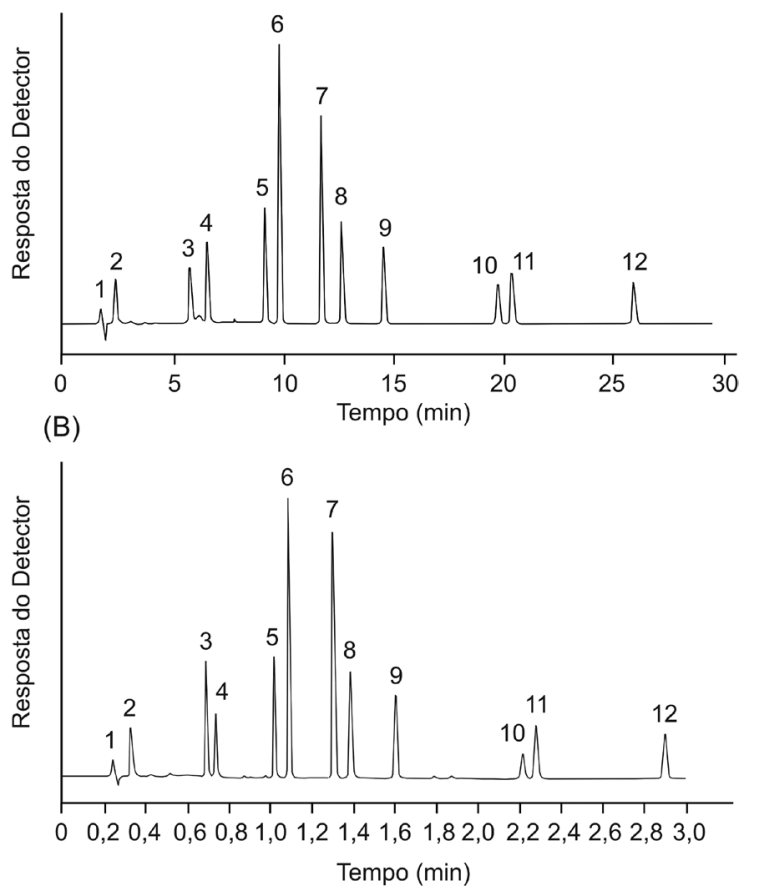

Figura 7. Cromatogramas ilustrativos da transferência de um método de separação no modo de eluição gradiente, desenvolvido para CLAE (A) e transferido para CLUE (B) para a separação de uma formulação farmacêutica. (A) Condições Cromatográficas: Coluna XBridge C18 (150 x 4,6 mm, 5 нm), FM: A-0,01\% de ácido fórmico em $\mathrm{H}_{2} \mathrm{O}$ e $\mathrm{B}-0,01 \%$ de ácido fórmico em acetonitrila:água $(50: 50 \mathrm{v} / \mathrm{v})$, vazão da $F M: 1000 \mu \mathrm{L} / \mathrm{min}$, gradiente: $T(A: B)=T_{0}(100: 0), T_{30}(40: 60), T_{31}(100: 0), T_{45}$ (100:0), volume de injeção: $20 \mu \mathrm{L}$, temperatura: $30^{\circ} \mathrm{C}$ e detecção UV. (B) Condições Cromatográficas: Coluna Acquity ${ }^{T M}$ BEH C18 (50 x 2,1 mm, 1,7 $\left.\mu \mathrm{m}\right), F M: A-0,01 \%$ de ácido fórmico em $\mathrm{H}_{2} \mathrm{O}$ e $\mathrm{B}-0,01 \%$ de ácido fórmico em acetonitrila:água (50:50 $v / v)$, vazão da $F M: 610 \mu \mathrm{L} / \mathrm{min}$, gradiente: $T(A: B)=T_{0}(100: 0), T_{3,4}(40: 60)$, $T_{3,5}$ (100:0), $T_{5,1}$ (100:0), volume de injeção: $1,4 \mu \mathrm{L}$, temperatura: $30^{\circ} \mathrm{C} e$ detecção UV. Adaptada da ref. 26
(A)

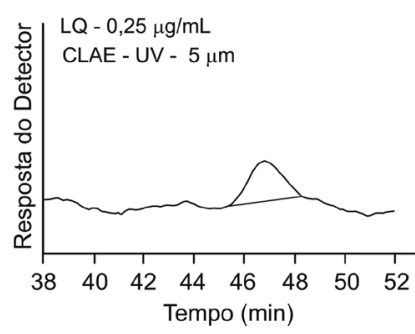

(B)

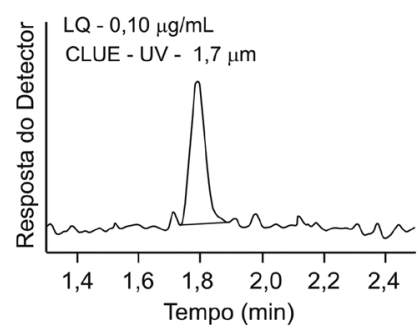

Figura 8. Medida dos LQ para a impureza II da primaquina. Condições Cromatográficas: (A) CLAE: FM: acetonitrila:água acidificada com 0,01\% de ácido trifluoracético $(25: 75 \mathrm{v} / \mathrm{v})$, vazão de FM: $1 \mathrm{~mL} / \mathrm{min}$, volume de injeção: $10 \mu \mathrm{L}$, detecção UV a $265 \mathrm{~nm}$ e temperatura: $35^{\circ} \mathrm{C}$. (B) CLUE: as mesmas condições que em (A) com exceção da vazão de FM: 0,5 mL/min e volume de injeção: 0,8 $\mu$ L. Adaptada da ref. 21

de fase móvel proporcionou um curto tempo de análise, enquanto que o uso de velocidades lineares de fase móvel intermediárias proporcionou um aumento na detectabilidade. A maior rapidez nas análises apresentou a vantagem de menor consumo de argônio.

Além dos trabalhos descritos acima, outros foram realizados empregando CLUE, como, por exemplo, determinação de lactonas, ${ }^{23}$ to- 
xinas marinhas, ${ }^{45}$ cianobactérias, ${ }^{46}$ drogas veterinárias, ${ }^{47}$ saponinas, ${ }^{12,48}$ enzimas $^{49}$ e metabonomas, ${ }^{50}$ o que comprova que a cromatografia líquida de ultra eficiência vem sendo avaliada e empregada em diversas áreas.

\section{CONCLUSÃO}

A cromatografia líquida de ultra eficiência é uma técnica bastante recente, que já está sendo utilizada em análises de rotina em diversas áreas. Esta rápida difusão está relacionada principalmente à disponibilidade da instrumentação totalmente desenvolvida e adequada para as necessidades do emprego de partículas $\leq 2 \mu \mathrm{m}$ e altas pressões e de materiais de recheio, como as partículas híbridas, que possuem desempenho cromatográfico superior às partículas de sílica pura.

Com base nos desenvolvimentos recentes, não resta dúvida que as análises realizadas utilizando-se CLUE são muito mais rápidas, na ordem de poucos minutos, que o consumo de solventes é bem menor, que as eficiências alcançadas são mais elevadas, que a detectabilidade é de 2-3 vezes maior, quando comparadas às análises realizadas utilizando-se CLAE. Além disso, a transferência de um método já desenvolvido por CLAE para CLUE pode ser facilmente realizada, deixando de ser um fator limitante para o emprego da CLUE.

Apesar de todas estas vantagens, poucos estudos foram realizados para avaliar o tempo de vida das colunas que são submetidas a condições extremas de pressão e o custo de manutenção de um equipamento que possui uma série de particularidades e opera em altas pressões. Além disso, a tecnologia das colunas de CLUE, que sejam capazes de gerar o máximo desempenho cromatográfico, requer novos desenvolvimentos neste campo.

\section{AGRADECIMENTOS}

Os autores agradecem à FAPESP e ao CNPq pelo apoio financeiro.

\section{REFERÊNCIAS}

1. Nguyen, D. T. T.; Guillarme, D.; Rudaz, S.; Veuthey, J. L.; J. Sep. Sci. 2006, 29, 1836.

2. Majors, R. E.; LCGC North Am. 2005, 23, 1248.

3. Neue, U.D.; Kele, M.; J. Chromatogr., A 2007, 1149, 236.

4. Kovalczuk, T.; Jech, M.; Poustka, J.; Hajslová, J.; Anal. Chim. Acta 2006, 577,8

5. Guillarme, D.; Nguyen, D. T. T.; Rudaz, S.; Veuthey, J. L.; J. Chromatogr., A 2007, 1149, 20.

6. Nguyen, D. T. T.; Guillarme, D.; Rudaz, S.; Veuthey, J. L.; J. Chromatogr., A 2006, 1128, 105.

7. Ikegami, T.; Tanaka, N.; Curr. Opin. Chem. Biol. 2004, 8, 527.

8. Wu, R.; Hu, L.; Wang, F.; Ye, M.; Zou, H.; J. Chromatogr., A 2008, no prelo.

9. Villiers, A.; Lestremau, F.; Szucs, R.; Gélébart, S.; David, F.; Sandra, P.; J. Chromatogr., A 2006, 1127, 60.

10. Wren, S. A. C.; Tchelitcheff, P.; J. Chromatogr., A 2006, 1119, 140.

11. Billen, J.; Guillarme, D.; Rudaz, S.; Veuthey, J. L.; Ritchie, H.; Grady, B.; Desmet, G.; J. Chromatogr., A 2007, 1161, 224.

12. Nováková, L.; Solichová, D.; Solich, P.; J. Sep. Sci. 2006, 29, 2433.

13. Nováková, L.; Matysová, L.; Solich, P.; Talanta 2006, 68, 908.

14. Swartz, M. E.; J. Liq. Chromatogr. Relat. Technol. 2005, 28, 1253.

15. Nguyen, D. T. T.; Guillarme, D.; Heinisch, S.; Barrioulet, M. P.; Rocca, J. L.; Rudaz, S.; Veuthey, J. L.; J. Chromatogr., A 2007, 1167, 76.

16. Bidlingmeyer, B. A.; Hooker, R. P.; Lochmuller, C. H.; Rogers, L. B.; Sep. Sci. 1969, 4, 439.
17. Jerkovich, A. D.; Mellors, J. S.; Jorgenson, J. W.; LCGC Eur. 2003, 16, 20.

18. Wu, N.; Lippert, J. A.; Lee, M. L.; J. Chromatogr, A 2001, 911, 1.

19. Dong, M. W.; LCGC North Am. 2007, 25, 656

20. Swartz, M. E.; LCGC North Am. Suppl. S 2005, Suppl. S, 8.

21. Dongre, V. G.; Karmuse, P. P.; Rao, P. P.; Kumar, A.; J. Pharm. Biomed. Anal. 2008, 46, 236.

22. Guan, J.; Lai, C. M.; Li, S. P.; J. Pharm. Biomed. Anal. 2007, 44, 996.

23. Englmann, M.; Fekete, A.; Kuttler, C.; Frommberger, M.; Li, X.; Gebefugi, I.; Fekete, J.; Kopplin, P. S.; J. Chromatogr., A 2007, 1160, 184.

24. Guillarme, D.; Nguyen, D. T. T.; Rudaz, S.; Veuthey, J. L.; Eur. J. Pharm. Biopharm. 2007, 66, 475.

25. Pedraglio, S.; Rozio, M. G.; Misiano, P.; Reali, V.; Dondio, G.; Bigogno, C.; J. Pharm. Biomed. Anal. 2007, 44, 665.

26. Guillarme, D.; Nguyen, D. T. T.; Rudaz, S.; Veuthey, J. L.; Eur. J. Pharm. Biopharm. 2008, 68, 430.

27. Apollonio, L. G.; Pianca, D. J.; Whittall, I. R.; Maher, W. A.; Kyd, J. M.; J. Chromatogr, B: : Anal. Technol. Biomed. Life Sci. 2006, 836, 111.

28. Chervet, J. P.; Ursem, M.; Anal. Chem. 1996, 68, 1507.

29. Vissers, J. P. C.; Ru, A. H.; Ursem, M.; Chervet, J. P.; J. Chromatogr., A 1996, 746,1

30. Grumbach, E. S.; Wheat, T. E.; Kele, M.; Mazzeo, J. R.; LCGC Eur. Suppl. S 2005, 44.

31. Nováková, L.; Solich, P.; J. Chromatogr., A 2005, 1088, 24.

32. King, S.; Stoffolano, P. J.; Robinson, E.; Eichhold, T. E.; Hoke, S. H.; Barker, T. R.; Richardson, E. C.; Wehmeyer, K. R.; LCGC North Am. Suppl. S 2005, 36.

33. Kaufmann, A.; Butcher, P.; Rapid Commun. Mass Spectrom. 2005, 19 , 3694.

34. HPLC calculator v.2.0, http://www.unige.ch/sciences/pharm/fanal/lcap/ divers/downloads.php, acessada em Fevereiro 2008.

35. Hordern, B. K.; Dinsdale, R. M.; Guwy, A. J.; Talanta 2008, 74, 1299.

36. Storbeck, K. H.; Kolar, N. W.; Sander, M.; Swart, A. C.; Anal. Biochem. 2008, 372, 11.

37. Hordern, B. K., Dinsdale, R. M.; Guwy, A. J.; J. Chromatogr., A 2007, 1161, 132.

38. Chen, L.; Qin, F.; Ma, Y.; Li, F.; J. Chromatogr., B: Anal. Technol. Biomed. Life Sci. 2007, 855, 255.

39. Farré, M.; Kuster, M.; Brix, R.; Ruibio, F.; Alda, M. J. L.; Barceló, D.; J. Chromatogr., A 2007, 1160166.

40. Hsieh, Y.; Duncan, C. J. G.; Lee, S.; Liu, M.; J. Pharm. Biomed. Anal. 2007, 44, 492.

41. Li, R.; Dong, L.; Huang, J.; Anal. Chim. Acta 2007, 546, 167.

42. Leandro, C. C.; Hancock, P.; Fussell, R. J.; Keely, B. J.; J. Chromatogr., A 2006, 1103, 94.

43. Leandro, C. C.; Hancock, P.; Fussell, R. J.; Keely, B. J.; J. Chromatogr, A 2007, 1144, 161.

44. Bendahl, L.; Hansen, S. H; Gammelgaard, B.; Sturup, S.; Nielsen, C.; J. Pharm. Biomed. Anal. 2007, 44, 648.

45. Fux, E.; McMillan, D.; Bire, R.; Hess, P.; J. Chromatogr, A 2007, 1157, 273.

46. Wang, J.; Pang, X.; Ge, F.; Ma, Z.; Toxicon 2007, 49, 1120.

47. Kaufmann, A.; Butcher, P.; Maden, K.; Widmer, M.; Anal. Chim. Acta 2007, 586, 13.

48. Wang, X.; Zhao, T.; Gao, X.; Dan, M.; Zhou, M.; Jia, W.; Anal. Chim. Acta 2007, 594, 265.

49. Yang, F. Q.; Guan, J.; Li, S.;P.; Talanta 2007, 73, 269.

50. Lenz, E. M.; Williams, R. E.; Sidaway, J.; Smith, B. W.; Plumb, R. S.; Johnson, K. A.; Shockcor, J.; Stumpf, C. L.; Granger, J. H.; Wilson, I. D.; J. Pharm. Biomed. Anal. 2007, 44, 845. 